\title{
A PDP-12 interface for time-code generator/readers
}

\author{
STEPHEN WALKER and MARTIN REITE \\ Department of Psychiatry, University of Colorado Medical Center, Denver, Colorado 80262
}

\begin{abstract}
An interrupt-driven time-code reader interface for the Digital Equipment Corporation PDP-12 minicomputer is described.
\end{abstract}

Time-code generator/readers are versatile laboratory instruments that come in a variety of configurations. Useful wherever data collected in different formats need to be time locked, they are especially important in locating data on analog (e.g., FM instrumentation) tape for computer processing of various types.

One standard type of time code for such applications is the Inter Range Instrumentation Group (IRIG) format, which consists of the time frame (seconds, minutes, hour, day) encoded in binary-coded decimal (BCD) on a variabie-frequency carrier. In the IRIG-B format used in our laboratory, the carrier frequency is $1 \mathrm{kHz}$ and the entire time frame is repeated once each second. ${ }^{1}$ A time-code generator produces the time code (e.g., in IRIG-B or other format) for recording on tape (a simultaneous slower code may be generated to record on paper records); the time-code reader

This program was supported by National Institute of Mental Health Grant MH19514 to Martin Reite. Martin Reite is also supported by National Institute of Mental Health Research Scientist Development Award, Type 2, 5K02 MH46335. accepts the time code from the tape in playback mode and translates it into a digital display output.

While the experimenter or computer operator can monitor the digital readout of time as a tape is replayed and select epochs for data analysis by hand, this is time consuming and cannot result in accuracy much better than $\pm 1 \mathrm{sec}$. A computer can also be programmed to read time code by digitizing the signal and subjecting it to logical analysis, but this is uneconomical from the standpoint of both software requirements and the fact that in the case of most laboratory computers, it ties up most of the machine.

In this paper we describe an interrupt-driven timecode reader interface for Digital Equipment Corporation (DEC) PDP-12 minicomputers. The interface, constructed from $M$ series modules, reads the digital display through a parallel $\mathrm{BCD}$ output on the time-code reader, allowing the processor to determine the time by means of three computer instructions.

Figure 1 shows a block diagram with interface between the time-code reader and the PDP-12 computer. The 1-pps signal from the time-code generator/reader

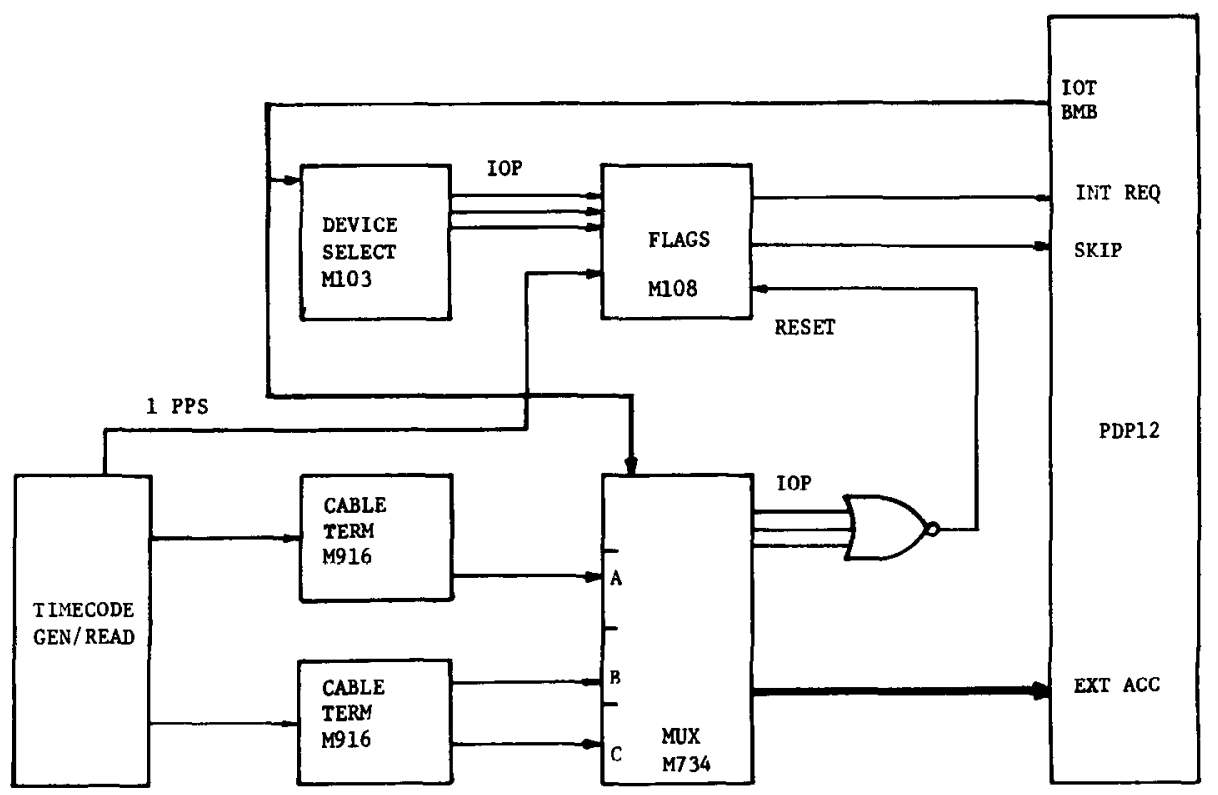

Figure 1. A block diagram of the time-code interface. 
goes high when the parallel BCD output data are stable, and sends an interrupt request (INT REQ) to the PDP-12 through the M108 flag module. The PDP-12 is alerted that a peripheral device wants service and polls all peripherals by testing each device-select module through the IOT BMB lines to see if it generated the interrupt. If the time-code reader/generator requests service, the device-select module (M103) sets a flag (SKIP) through the M108 flag module. The PDP-12 then enters a time-code generator/reader service routine. The PDP-12 uses the same IOT BMB lines to read A, B, or $C$ (defined below) into the processor's accumulator through the EXT ACC lines.

The MULTIPLEXOR (M734, called MUX) contains its own device-selector logic and can read any one of the three input words. Usual practice is to read all three in sequence on the occurrence of each interrupt. In this system, the $C$ input is the day of the year, the $B$ section is the hour and minute, and the A section is seconds and least significant digit of the minutes. The interrupt request is cleared by the NOR gate output to the flag module when reading any portion of the time.

The device-select section of the interface (M103) was assigned device number 36 and the reader (M734) was assigned device number 37 . These numbers are hard-wired by connecting the appropriate BMB lines to the address inputs of the respective modules. The operation codes are in Table 1.

The interface permits a tape to be played back faster than real-time. For example, at 8 times real-time playback, the processor is only interrupted 8 times/sec. Timing of data analysis epochs can be as precise as desired, within the limit of tape recorder speed control and the computer's internal clock.

Decoding software routines is relatively simple and permits considerable user versatility. We have written several for the PDP-12 that are available on request for the cost of duplication. Available routines include
Table 1

Interface Instruction Set

\begin{tabular}{ll}
$\begin{array}{c}\text { Operation } \\
\text { Code }\end{array}$ & \\
\hline 6361 & Skip if flag set \\
6362 & Enable interrupt \\
6364 & Disable interrupt \\
6371 & A read BCD second (+ LSD min), clear interrupt \\
6372 & B read BCD hours-minutes (- LSD), clear interrupt \\
6374 & C read BCD days, clear interrupt \\
\hline
\end{tabular}

TIME, a DIAL program that displays the date or time on a VR-14 display; START, a FORTRAN-callable RALF routine for initializing the reader interface and starting it; and STOP, a FORTRAN-callable RALF routine to stop the interface from interrupting the processor.

This interfacing technique of reading a parallel $\mathrm{BCD}$ signal through a digital input can be adapted for other types of computers. We are currently designing the time-code interface for PDP-11/03s by using a DRV11 parallel board. DEC-supplied FORTRAN extensions can read the time through standard subroutines (DRS, IDIR, or DINP).

\section{NOTE}

1. A general discussion of time keeping can be found in a pamphlet entitled Precision Timekeeping and Tape Search, published in 1974 by the Systron-Donner Corporation, 1 Systron Drive, Concord, California 94518, and in a pamphlet entitled IRIG Telemetry Standards, prepared by the EMR Telemetry Division of Electromechanical Research, Inc., of Sarasota, Florida (also published in 1966 as Document 106-66 by the Secretariat, Range Commanders Council, White Sands Missile Range, New Mexico 88002.

(Received for publication November 22, 1977 revision accepted May 12,1978 .) 